\title{
Acute Effect of Active and Passive Static Stretching on Elastic Modulus of the Hamstrings
}

(1) $\Theta \Theta$

\author{
Authors \\ Gakuto Nakao $^{1,2}$, Keigo Taniguchi ${ }^{3}$, Masaki Katayose ${ }^{3}$
}

\author{
Affiliations \\ 1 Graduate School of Health Sciences, Sapporo Medical \\ University, Sapporo, Japan \\ 2 Department of Rehabilitation, Ebetsu City Hospital, \\ Ebetsu, Japan \\ 3 Department of Physical Therapy, Sapporo Medical \\ University, Sapporo, Japan
}

\author{
Key words \\ muscle strain, flexibility, range of motion, \\ shear wave elastography \\ received $\quad 18.04 .2018$ \\ revised $\quad 09.08 .2018$ \\ accepted 11.08 .2018

\section{Bibliography} \\ DOI https://doi.org/10.1055/a-0733-6957 \\ ISSN 2367-1890

\section{Correspondence} \\ Mr. Gakuto Nakao, master \\ Graduate School of Health Sciences \\ Sapporo Medical University \\ South-3, West-17 \\ Chuo-Ku, Sapporo \\ 060-8556 Hokkaido \\ Japan \\ Tel.: + 81/11/611 2111 \\ gakunn.24@gmail.com
}

Sports Medicine International Open 2018; 2: E163-E170

(c) Georg Thieme Verlag KG Stuttgart · New York

\begin{abstract}
The purpose of this study was to investigate differences in the acute effects of passive knee extension (PKE) and active knee extension (AKE) stretching on the shear elastic modulus of the hamstrings. In 20 healthy men, maximum knee extension (maximum range of motion [ROM]) and shear elastic modulus of the hamstrings (biceps femoris long head, semitendinosus, semimembranosus) were measured before (Pre) and after (Post) AKE and PKE stretching. The maximum ROM during stretching was measured. In both stretching methods, maximum ROM increased and the shear elastic modulus decreased $(p<0.01)$, but no difference was found between the procedures. No significant difference was observed in the maximum ROM during stretching between the procedures $(p=0.06)$. The shear elastic modulus was significantly lower in the biceps femoris long head and semimembranosus than in the semitendinosus muscle $(p<0.05)$. Static stretching with PKE and AKE stretching showed an increase in maximum ROM and a decrease in hamstring elasticity, but no difference was found between the methods. Both stretching protocols were effective for reducing elasticity of the biceps femoris long head and semimembranosus.
\end{abstract}

\section{Introduction}

The incidence of muscle strain is reported to be the highest in the hamstrings $[7,17]$. The hamstrings are composed of the biceps femoris long head (BF-lh)/short head (BF-sh), semitendinosus (ST), and semimembranosus (SM). Among them, the occurrence of BF-lh is the highest $[2,4,5]$. In addition, hamstring strain easily recurs, and chronicity often leads to long-term withdrawal from competitive sport [44]. Therefore, preventing hamstring strain and its recurrence is important.
Lack of hamstring flexibility (high stiffness) may result in muscle strain $[1,2,28]$. Static stretching (SS) has been used to increase flexibility [26]. As stretching methods for the hamstrings, passive knee extension (PKE) stretching for passively extending the knee joint $[24,34]$ and active knee extension (AKE) stretching for actively extending the knee joint $[6,8]$ are frequently used.

Review papers on stretching have reported the immediate effect of AKE and PKE stretching, and both techniques are effective in increasing the maximum range of motion (ROM) [27, 33]. Changes 
in ROM are caused by neurophysiological factors and mechanical factors, such as musculotendinous stiffness [11, 12, 24, 26, 37, 38]. Previous studies have examined the effect of passive SS on neurophysiological properties using evoked electromyography, and reported that passive SS can suppress muscle tone [11, 37, 38]. The amplitude of the $\mathrm{H}$ wave and the maximum amplitude ratio to the $M$ wave (Hmax / Mmax) are representative indicators of evoked electromyography, and the change in amplitude of the $\mathrm{H}$ wave reflects the excitability of the anterior horn cell of the spinal cord [14]. As the ratio of $\mathrm{Hmax} / \mathrm{Mmax}$ reportedly decreases after passive SS [37], thus resulting in suppressed muscle tone from passive SS. This neurophysiological effect causes Ib suppression by the Golgi tendon organ from muscle stretching and suppresses excitation of motor neurons of the target muscle. By contrast, the stretching mechanism of active SS has a neurophysiological effect that causes reciprocal suppression (la suppression) by contraction of antagonist muscle groups; moreover, it suppresses excitability of motor neurons of target muscles [10,12,14,19]. Previous studies have examined the stretching effect of reciprocal suppression $[10,12,19]$ and reported that passive torque at the extended position decreases [10]; furthermore, the electromyographic amplitude decreased after stretching [12] due to contraction of the antagonist muscle of the hamstrings. Therefore, the mechanisms by which PKE and AKE affect neurophysiological properties are different. However, a previous comparison between passive and active SS did not clearly show differences in the effects of stretching, because of differences between studies in stretching position and stretching time [29, 42]. Nishikawa et al. compared the stretching effect of AKE and PKE stretching by unifying stretching position and stretching time [33], and found that the maximum ROM increased more in PKE than in AKE stretching. However, when evaluating the ROM change, it is impossible to determine the factors that cause the ROM change, and the details of stretching mechanisms remain unknown [15].

In recent years, the shear elastic modulus of muscles, measured using ultrasonic shear wave elastography, has been reported to be highly correlated with passive tension $[19,24]$; thus, as the shear elastic modulus increases, the muscle is increasingly stretched. Le Sant et al. reported that shear elastic modulus at extension was different between the hamstrings [22]. This suggests that the mechanical properties of muscles may be involved in the risk of muscle injury [28]. Several studies have examined the influence of stretching on mechanical stress $[30,41]$. Umegaki et al. and Miyamoto et al. reported that shear elastic modulus and maximum ROM before and after PKE stretching were measured for the hamstrings using ultrasonic shear wave elastography $[29,40]$. The increase of the maximum ROM and the decrease of the shear elastic modulus were observed after stretching. This finding suggests that the increase in maximum ROM due to stretching in PKE stretching may be due to a decrease in the shear modulus of the muscle. PKE stretching is known to increase the maximum ROM, but although AKE stretching is also frequently used as a stretching technique, the effect of AKE stretching on mechanical stress is not clear. In addition, it is unclear whether AKE or PKE stretching is a more effective procedure for decreasing the shear modulus of the muscles for individual muscles of the hamstrings. In this study, we aimed to investigate the acute effect of the difference in PKE and AKE stretch- ing on the shear elastic modulus of the hamstrings using shear elastic modulus as an index of stretching effect.

\section{Methods}

\section{Subjects}

Twenty healthy men (age, $24.0 \pm 4.1$ years; height, $170.5 \pm 4.4 \mathrm{~cm}$; weight, $64.0 \pm 5.1 \mathrm{~kg}$ ) volunteered for this study. Subjects with a history of neuromuscular disease or musculoskeletal injury involving the lower extremities were excluded. All subjects signed an informed consent form agreeing to participate in the study. The study conformed to the Declaration of Helsinki and was approved by the Ethics Committee of Sapporo Medical University (Approval number: 29-2-37). This study was also performed in accordance with the ethical standards of the International Journal of Sports Medicine [9].

\section{Experimental procedure}

A crossover design was used in this experiment. Two different stretching conditions (AKE stretching condition, PKE stretching condition) were performed for all subjects. PKE and AKE stretching were performed in random order on the subject's dominant leg. The dominant leg was determined as the leg used by the participant to kick a ball [16]. To avoid the stretching effect, stretching was carried out at random intervals of one day [35]. The maximum ROM and shear elastic modulus were measured and compared preand post-stretching conditions. During stretching, maximum ROM was also measured.

\section{SS protocol}

Two different stretching conditions (AKE stretching condition, PKE stretching condition) were performed on the subject's dominant leg. The starting stretching position was hip and knee joint angles of $90^{\circ}$ flexion in the supine position, and this position was fixed manually by the operator. The non-dominant leg and pelvis were fixed to the bed with a belt to avoid movement. The AKE stretching condition was achieved by actively extending the knee joint. For the AKE stretching, the subjects were instructed to extend the knee until the maximum tolerable stretch of the hamstring muscles was reached. During AKE stretching, the subjects were instructed to maintain the lower leg rotation angle in the neutral position, due to the possibility that the lower leg rotation angle could influence the stretching effect. The PKE stretching condition was achieved identically, except that the knee was passively extended by the examiner by manual operation. As for the extension strength in AKE and PKE stretching, the optimum intensity was determined when the subject felt that the extension was maximized without discomfort or pain [31]. The start time of stretching was recorded when the subject felt that the optimum intensity was reached. Stretching was performed at a slow speed $(5 \%)$ to prevent muscle contraction due to pain (stretch reflex). A previous study reported that $>2$ min of stretching decreases muscle stiffness [32]. Therefore, in this study, PKE and AKE stretching were performed for $30 \mathrm{~s}$ and repeated for five sets, and the interval between each set was 20 s. To exclude the influence of pre-stretching and exercise, all subjects were instructed not to perform stretching and exercise just before the measurement. 


\section{Measurement of the maximum ROM}

The starting position was hip joint and knee flexion of $90^{\circ}$. The subject was placed in a supine position on the bed. The opposite lower limbs and pelvis were firmly fixed with a belt to prevent pelvic tilt due to hamstring extension. Because the hip flexion angle may affect the knee joint extension angle, it was manually fixed. Maximum ROM was measured using a TODAI type goniometer (Sakai Medical, Tokyo, Japan). The maximum ROM was defined as the strength without discomfort or pain when the knee joint was elongated passively. The final angle was measured in $1^{\circ}$ increments. The maximum ROM measurement of all subjects was measured by the same examiner. The number of measurements was set to 2 , and the high value was recorded. Considering that the difference in knee joint extension angle may influence extension strength, the maximum ROM during stretching was measured. The measurement was performed at the last two of the 5 sets, and the high value was recorded.

\section{Measurement of the shear elastic modulus}

To measure shear elastic modulus, the subjects were placed in a supine position with the hip at $90^{\circ}$ flexion, knee at $45^{\circ}$ flexion, and the pelvis secured with a belt ( $\vee$ Fig. 1$)$. This position was fixed using a dynamometer (Biodex system 4.0; Sakai Medical Instruments, Tokyo, Japan). Shear elastic modulus was measured with ultrasound shear wave elastography (Aixplorer 6.0, MSK mode; SuperSonic Imagine, Aix-en-Provence, France) with a linear array probe $(50 \mathrm{~mm}$, 4-15 Hz, SL15-4; Supersonic Imagine, Aix-en-Provence, France). Each ultrasound probe was placed over the muscle belly of BF-lh, $\mathrm{ST}$, and SM in a random order across subjects. The probe was placed parallel to the muscle fibers and was held gently over the skin without applying pressure to the tissues underneath. An acoustic gel was used as an interface between the probe and the skin. The measurement site was located longitudinally at $50 \%$ of the lower leg length measured from the ischial tuberosity to the medial epicondyle of the thighbone for the ST and SM and to the lateral epicondyle of the thighbone for the BF-lh. To distinguish between ST and
SM muscles (medial muscle), tendinous inscription of ST muscle was confirmed [43]. The points were confirmed beforehand by palpation and B-mode image. After finding the clearest image, the location of the probe was marked with permanent ink. With regard to the measurement principle of the shear elastic modulus, Young's modulus was quantified in $\mathrm{kPa}$ of the region of interest (ROI) of $15 \times 15 \mathrm{~mm}^{2}$ in each muscle region based on the shear wave propagation velocity, C ( $\triangleright$ Fig. 2). For each pixel of the ROI, Young's modulus, E, was calculated as follows:

$\mathrm{E}=3 \rho c^{2}$

where $\rho$, muscle mass density, is assumed to be constant $\left(1000 \mathrm{~kg} / \mathrm{m}^{3}\right)$ in human soft tissues. Within the ROI above, a circular area $(10 \mathrm{~mm}$ in diameter) that included clearer fascicle without evident connective tissues was chosen. The spatial average of Young's modulus of

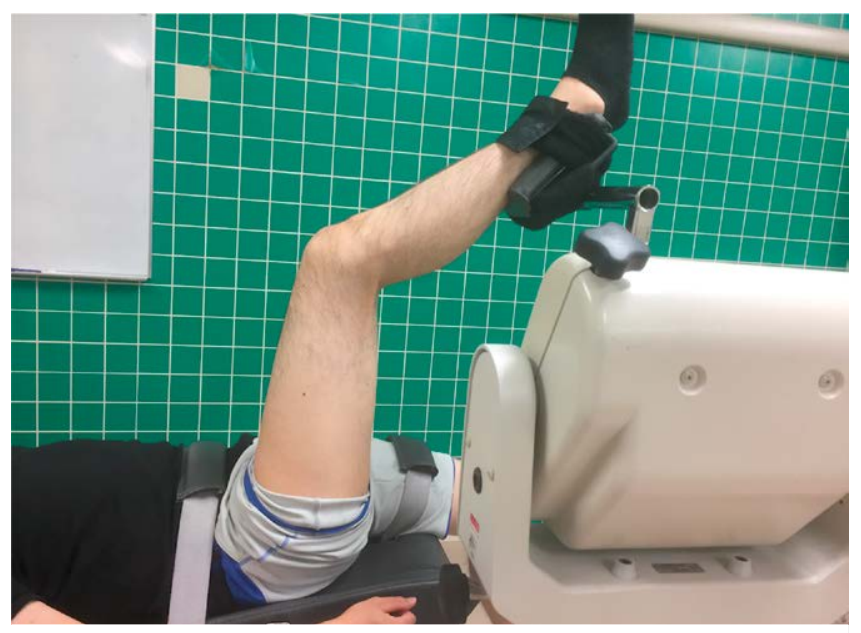

Fig. 1 Position of measurement of the shear elastic modulus (hip at $90^{\circ}$ flexion and knee at $45^{\circ}$ flexion).

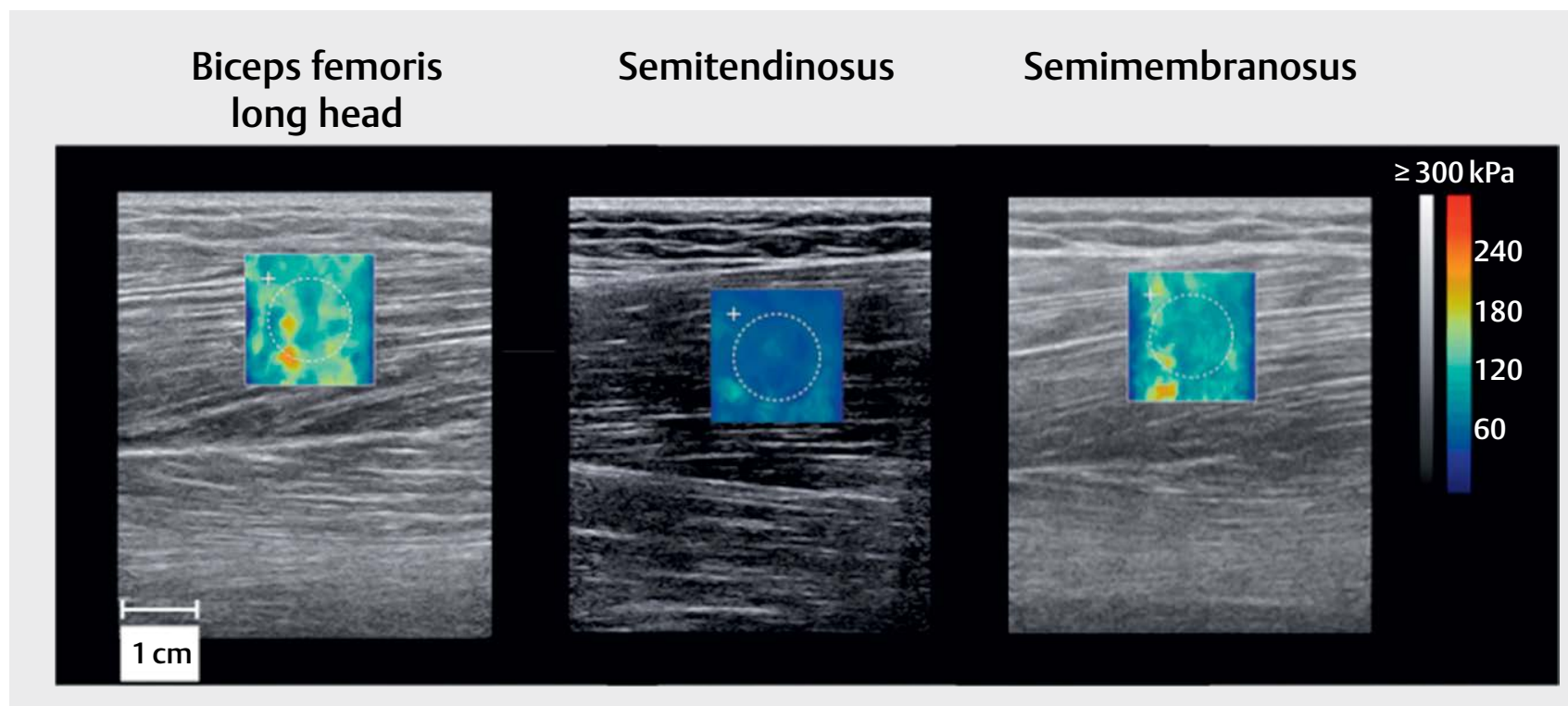

- Fig. 2 Typical example of shear elastic modulus measurement of the biceps femoris long head, semitendinosus, and semimembranosus. 
the selected circular area was calculated in $\mathrm{kPa}$. The device yields Young's modulus by applying the constant 3 based on the assumed isotropic nature of soft tissues, whereas skeletal muscle cannot be assumed to be isotropic [39]. Therefore, shear elastic modulus was used by dividing the obtained Young's modulus by 3 . The shear elastic modulus is an index of stiffness. The muscles are hardened by being elongated and possess the property of becoming less distorted. In previous studies [20,25], the correlation between shear elastic modulus and elongation of muscles is highly correlated so that the greater the shear elastic modulus, the more stretched the muscle. The shear elastic modulus of each muscle was measured twice. The mean value of the two measurements for each muscle was used for statistical analyses.

\section{Statistical analysis}

Measurements of the maximum ROM and shear elastic modulus of each muscle were performed by the same examiner. In twenty healthy men, within-session intra-rater reliability of maximum ROM and shear elastic modulus of each muscle were assessed using the intraclass correlation coefficient (ICC $[1,1]$ ) with a $95 \%$ confidence interval (CI). In addition, because AKE and PKE stretching were performed on different days, the day-to-day reliability was also examined for maximum ROM and shear elastic modulus. The day-to-day reliability interval was one day. Two-way repeated measures analysis of variance (ANOVA) using the factors of procedure (AKE stretching condition and PKE stretching condition) and test time (pre and post) was used to analyze interaction effects for the maximum ROM. A paired t-test was performed to determine the differences in the maximum ROM during stretching. Two-way repeated measures ANOVA using procedure and test time was used to analyze interaction effects on each muscle for the shear elastic modulus at $90^{\circ}$ of hip flexion and $45^{\circ}$ of knee joint flexion. To examine the stretching effect between muscles in both types of stretching, the reduction rate of the shear elastic modulus pre- and poststretching was calculated, and two-way repeated measures ANOVA using procedure and muscle was used to analyze interaction effects. The reduction rate was calculated by (Pre - Post)/Pre $\times 100$ as the shear elastic modulus in the pre- and post-stretching. When a significant main effect of muscle was observed, a post-hoc analysis with Bonferroni correction was performed where appropriate. The level of statistical significance was set at $p=0.05$. Data were reported as mean and standard error (mean \pm SE). Statistical analyses were performed using SPSS (version 19.0, SPSS Japan Inc., Tokyo, Japan).

\section{Results}

\section{Within-session intra-rater reliability and day-to-day reliability of maximum ROM and shear elastic modulus of each muscle}

Within-session intra-rater reliability and day-to-day reliability of maximum ROM and shear elastic modulus of each muscle are shown in $>$ Table 1. Regarding shear elastic modulus, the $\operatorname{ICC}(1,1)$ of within-session intra-rater reliability ranged from 0.98 to 0.99 for the shear elastic modulus of BF-lh, from 0.95 to 0.99 for the shear elastic modulus of ST, and from 0.98 to 0.99 for the shear elastic modulus of SM. The ICC $(1,1)$ of day-to-day reliability was 0.84 for the shear elastic modulus of BF-lh, 0.62 for the shear elastic modulus of ST, and 0.82 for the shear elastic modulus of SM. Regarding the maximum ROM, the ICC $(1,1)$ of within-session intra-rater reliability ranged from 0.98 to 0.99 . The ICC $(1,1)$ of day-to-day reliability was 0.88 for the maximum ROM.

\section{Maximum ROM}

The means of the maximum ROM are shown in > Fig. 3. No interaction (procedure $\times$ test time) was observed in the maximum ROM pre- and post-AKE and PKE stretching $(F=0.147, p=0.706)$. The

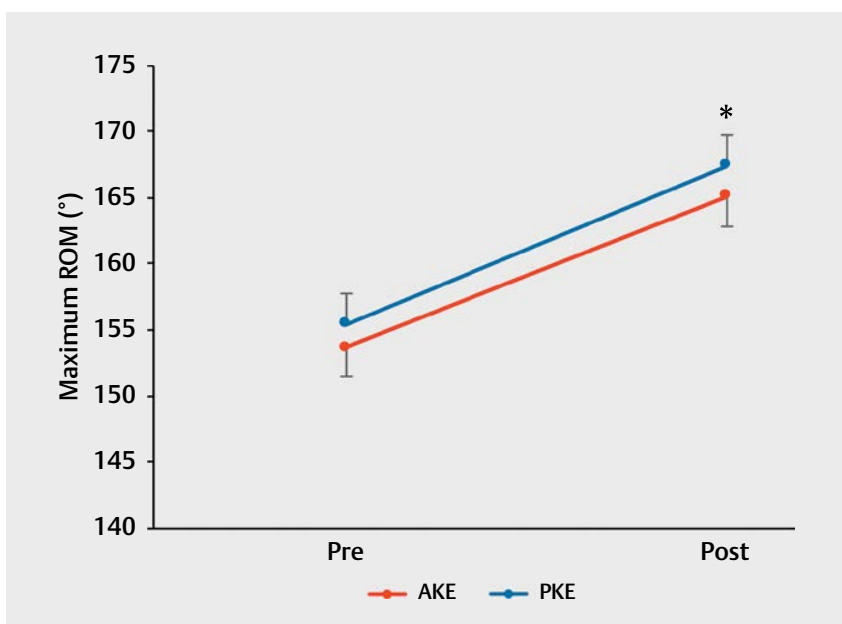

- Fig. 3 Maximum ROM pre- and post-AKE and PKE. Values are shown as mean \pm SE (standard error). AKE: active knee extension, PKE: passive knee extension, Pre: before stretching, Post: immediately after stretching. ${ }^{*}$ Significantly different between pre and post $(p<0.01)$

> Table 1 Within-session intra-rater reliability and day-to-day reliability of maximum ROM and shear elastic modulus of each muscle.

\begin{tabular}{|c|c|c|c|c|c|c|}
\hline & & \multicolumn{2}{|c|}{ AKE } & \multicolumn{2}{|c|}{ PKE } & \multirow{2}{*}{$\begin{array}{c}\text { Day- to day reliability } \\
\text { Pre only }\end{array}$} \\
\hline & & Pre & Post & Pre & Post & \\
\hline & & $\mathrm{ICC}_{1,1}(95 \% \mathrm{CI})$ & ICC $_{1,1}(95 \% \mathrm{Cl})$ & $\mathrm{ICC}_{1,1}(95 \% \mathrm{Cl})$ & $\mathrm{ICC}_{1,1}(95 \% \mathrm{Cl})$ & ICC $_{1,1}(95 \% \mathrm{Cl})$ \\
\hline \multirow[t]{3}{*}{ Shear elastic modulus } & BF-Ih & $0.99(0.99-0.99)$ & $0.99(0.99-0.99)$ & $0.98(0.95-0.99)$ & $0.98(0.97-0.99)$ & $0.84(0.66-0.93)$ \\
\hline & ST & $0.99(0.98-0.99)$ & $0.98(0.97-0.99)$ & $0.95(0.89-0.98)$ & $0.95(0.88-0.98)$ & $0.62(0.27-0.83)$ \\
\hline & $\mathrm{CM}$ & $0.99(0.99-0.99)$ & $0.98(0.96-0.99)$ & $0.99(0.99-0.99)$ & $0.99(0.98-0.99)$ & $0.82(0.62-0.92)$ \\
\hline Maximum ROM & & $0.99(0.98-0.99)$ & $0.99(0.97-0.99)$ & $0.99(0.98-0.99)$ & $0.99(0.97-0.99)$ & $0.88(0.73-0.95)$ \\
\hline
\end{tabular}


main effect was observed in test time $(F=57.530, p=0.001)$, but was not observed in procedure $(F=3.654, p=0.071)$. The maximum ROM significantly increased post-stretching compared with prestretching (pre, $154.5 \pm 2.3^{\circ}$; post, $166.2 \pm 1.9^{\circ} ; p=0.001$ ). No significant difference was observed in the maximum ROM during the stretching between the procedures (AKE stretching, 162.9 $\pm 2.1^{\circ}$; PKE stretching, $\left.166.3 \pm 2.2^{\circ} ; \mathrm{p}=0.06\right)$.

\section{Shear elastic modulus}

The means of the shear elastic modulus in each muscle are shown in $>$ Fig. 4. In all muscles, no interaction (procedure $\times$ test time) was observed in shear elastic modulus before and after AKE and PKE stretching (BF-lh, $F=0.369, p=0.551 ; S T, F=0.331, p=0.583$; $\mathrm{SM}, \mathrm{F}=0.17, \mathrm{P}=0.685)$. The main effect was recognized as test time (BF-lh, $F=35.62, p=0.001 ; S T, F=18.231$, $p=0.001 ; S M, F=27.773$, $\mathrm{P}=0.001)$, but was not recognized as procedure (BF-lh, $F=1.384$, $p=0.254 ; S T, F=2.846, p=0.108 ; S M, F=0.684, p=0.418)$. The shear elastic modulus was significantly lower post-stretching than pre-stretching (BF-lh pre, $39.7 \pm 3.7 \mathrm{kPa}$; post, $27.2 \pm 2.2 \mathrm{kPa}$, $\mathrm{p}=0.001$; ST pre, $28.9 \pm 1.7 \mathrm{kPa}$; post, $24.3 \pm 1.4 \mathrm{kPa}, \mathrm{p}=0.001 ; \mathrm{SM}$ pre, $50.4 \pm 5.2 \mathrm{kPa}$; post, $37.6 \pm 4.1 \mathrm{kPa}, \mathrm{p}=0.001)$. The results for the reduction rate of the shear elastic modulus before and after AKE and PKE stretching are shown in > Fig. 5. No significant main effect of procedure $(F=0.005, p=0.946)$ or interaction of procedure and muscle $(F=0.524, p=0.601)$ was observed in the reduction rate of the shear elastic modulus, whereas a significant main effect was recognized as muscle $(F=4.169, p=0.032)$. With a main effect of muscle, the reduction rate of the shear elastic modulus pre- and post-stretching was significantly higher in the BF-lh $(27.8 \pm 3.3 \%)$ and SM muscles $(24.1 \pm 3.5 \%)$ than in the ST muscle $(13.8 \pm 3.5 \%)$ when averaged across procedure.

\section{Discussion}

In this study, the stretching effect of AKE and PKE stretching showed an increase in the maximum ROM and a decrease in muscle elastic- ity of the hamstrings, but no difference was found between the procedures on reduction of muscle elasticity. In addition, the reduction rate of the shear modulus pre- and post-stretching was higher in the BF-Ih and SM than in the ST. Some studies have reported the comparison of the maximum ROM between AKE and PKE stretching and the stretching effect of shear modulus with PKE stretching elasticity. To our knowledge, the present study is the first to report on the stretching effect of the shear elastic modulus of the hamstrings in AKE stretching and compare the stretching effect of PKE and AKE stretching by shear elastic modulus.

\section{Within-session intra-rater reliability and day-to-day reliability of maximum ROM and shear elastic modulus of each muscle}

In this study, repeatability of measuring maximum ROM and shear elastic modulus of BF-Ih, ST, and SM was evaluated using $\operatorname{ICC}(1,1)$. The repeatability was assumed to be good if ICC was above 0.75 , moderate if ICC was between 0.50 and 0.75 , and poor if ICC was below 0.50 , using previously established criteria [38]. Regarding within-session intra-rater reliability, the ICC in this study was well above 0.75 (0.95-0.99) and the data in this study were thus considered reliable. These results agree with those of a previous study [40]. Regarding day-to-day reliability, shear elastic modulus of SM, BF-Ih, and maximum ROM showed good reliability, but ST showed moderate reliability. Ultrasonic shear wave elastography is a method for quantitatively evaluating the stiffness of a tissue by generating a shear wave in the tissue and measuring its propagation velocity. When the compression by the ultrasonic probe in contact with the body surface becomes strong, the tissue becomes hard [21]. Therefore, appropriate operation of the probe is required. In this study, the examiner received sufficient training before measurement to prevent body surface compression. However, when the probe was fixed to the ST, the examiner often experienced the probe slipping from the ST and it was difficult to fix the probe. The anatomical structure of the hamstrings muscle is complicated, and the ST has a smaller cross-sectional area than does the SM or BF-Ih $[18,43]$.

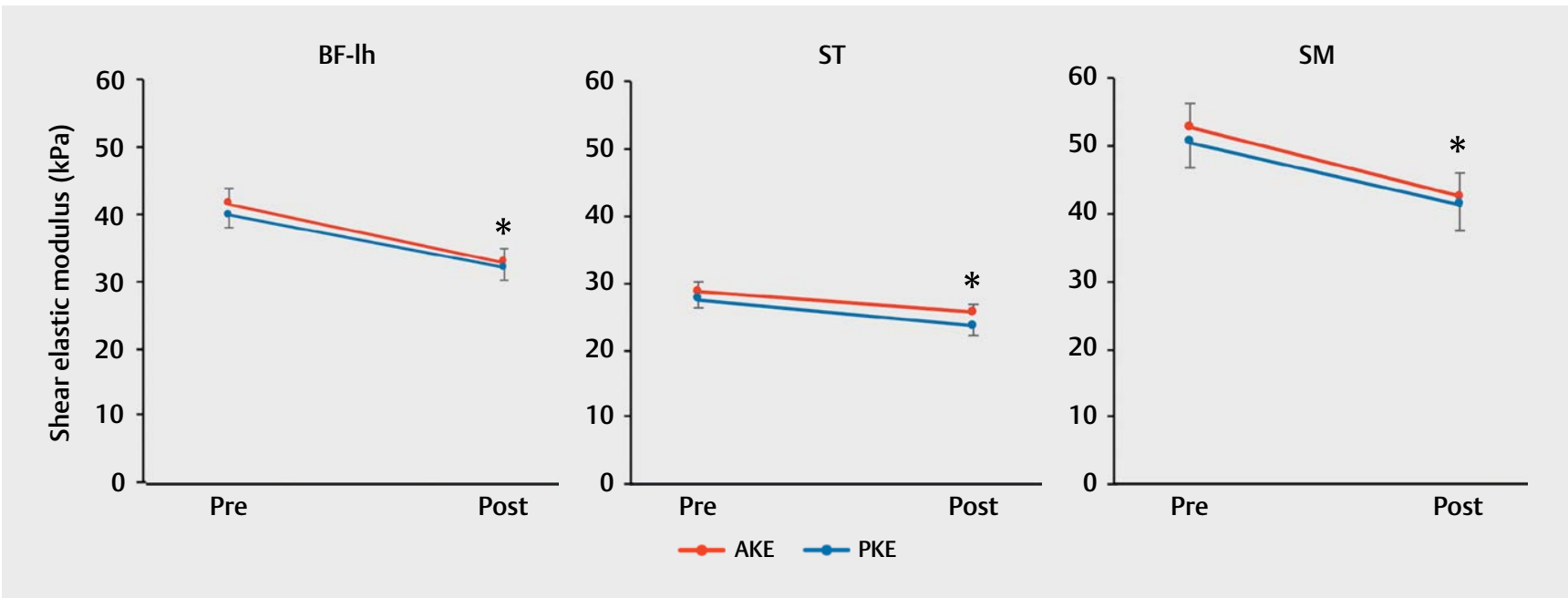

-Fig. 4 Shear elastic modulus of the BF-Ih, ST, SM pre- and post-AKE, and PKE. Values are shown as mean \pm SE (standard error). AKE: active knee extension, PKE: passive knee extension, Pre: before stretching, Post: immediately after stretching, BF-lh: biceps femoris long head, ST: semitendinosus, SM: semimembranosus. *Significantly different between pre and post $(p<0.01)$. 


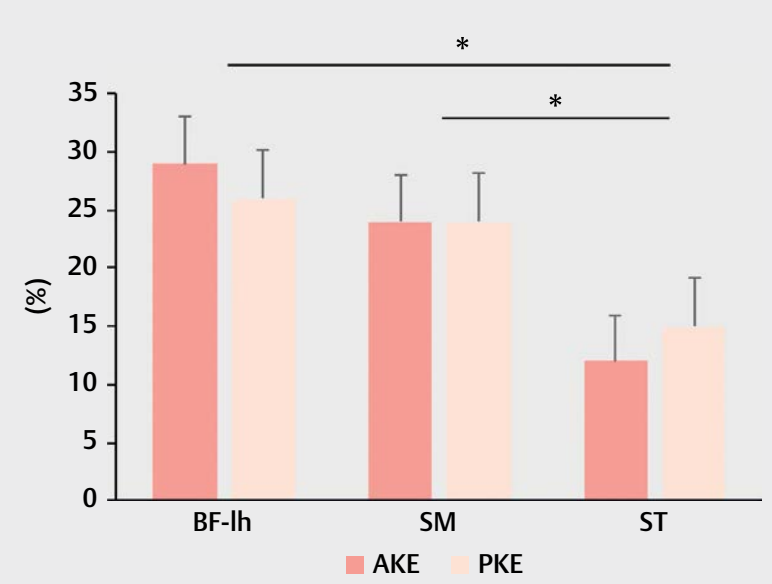

-Fig. 5 The reduction rate of the shear elastic modulus between hamstring muscles in AKE and PKE. AKE: active knee extension, PKE: passive knee extension, BF-lh: biceps femoris long head, ST: semitendinosus, SM: semimembranosus. *Main effect of muscle. Significantly higher than semitendinosus $(p<0.05)$.

In muscles with a small cross-sectional area such as ST, there was a possibility that the pressure of the probe became strong so as not to slip the probe from the skin.

\section{Stretching effect on the maximum ROM}

The maximum ROM increased with both procedures after stretching. However, no difference in maximum ROM was observed between both procedures. In contrast to our findings, Nishikawa et al. reported that PKE stretching is more effective in increasing maximum ROM than AKE stretching [33]. The two possible differences in the method between the studies are as follows. First, we evaluated ROM using a passive knee extension test, which passively extends the knee joint, whereas Nishikawa et al. evaluated ROM using an active knee extension test, which actively extends the knee joint. Evaluation of ROM is generally divided into evaluation of passive exercise to evaluate flexibility of soft tissue [27] and evaluation of active exercise to evaluate agonist muscle strength and coordination of exercise [40]. Therefore, the method of choice for evaluating ROM depends on the purpose. Because the purpose of this study was to investigate the stretching effect on the elasticity of the muscles, evaluation to stretch the knee joint passively was considered, that is, stretching evaluation using a passive knee extension test was reasonable. Second, we performed a within-subject comparison, whereas Nishikawa et al. performed a between-subject comparison. The amount and structural characteristics of the collagen fibers of the fascia affect the extensibility of the muscles, and there are individual differences among them [36]. Therefore, a comparison of subjects may yield different physical characteristics affecting the extensibility among individuals. In this study, differences in ROM before stretching and physical characteristics had a minor effect on stretching.

\section{Stretching effect on shear elastic modulus}

The shear elastic modulus decreased with both procedures after stretching. However, no difference in shear elastic modulus of the BF-lh, ST, and SM was observed between both procedures. Previ- ous studies $[30,32,41]$ reported a decrease in muscle elasticity after stretching, which is in agreement with our results. Like the influence of the connective tissue on the extensibility of skeletal muscle, passive tension decreases with muscle fiber bundle. This removes the connective tissue component including fascia as much as possible from the intact muscle fiber bundle. Thus, the passive tension may be involved in the connective tissue including the fascia [36]. Because the shear elastic modulus of the muscle measured using ultrasonic shear wave elastography is strongly correlated with the passive tension $[20,25]$, the decrease in elasticity of the muscle due to stretching (reduction in passive tension) may be due to the change in connective tissue such as the fascia. Detailed studies on the mechanism of active stretching have not been conducted in previous studies $[10,12,19]$. In this study, AKE stretching showed a decrease in muscle elasticity. AKE stretching produces a stretching effect not only for muscle tone relaxation of hamstrings due to reciprocal suppression but also for muscle structure.

With regard to the shear elastic modulus pre- and post-stretching between hamstrings, a previous study reported that SM showed the most elongation [41]. By contrast, we found no difference between BF-lh and SM. Umegaki et al. reported a stretching time of $5 \mathrm{~min}$ ( $1 \mathrm{~min} \times 5$ sets), whereas our study had a stretching time of 2 min $30 s$ ( $30 s \times 5$ sets). Connective tissues, such as muscles, have time-dependent mechanical properties, and their characteristics appear during stretching [23]. Thus, differences in stretching times may have influenced the results.

With regard to the factor that the rate of decrease of the shear modulus of elasticity differs between muscles, differences in hamstring anatomical features may be involved. According to cadaveric anatomical studies [18], the physiological cross-sectional area is larger for the SM $\left(18.21 \pm 4.21 \mathrm{~cm}^{2}\right)$ and BF-lh $\left(12.68 \pm 2.58 \mathrm{~cm}^{2}\right)$ than for the ST $\left(5.39 \pm 1.59 \mathrm{~cm}^{2}\right)$. Recently, Miyamoto et al. examined whether passive muscle shear modulus is related to muscle cross-sectional area in the hamstrings. As a result, they suggested that there were no significant correlations between shear modulus and cross-sectional area in each or overall hamstrings [13]. On the other hand, it is reported that the fascicle length is shorter in the SM $(5.43 \pm 0.87 \mathrm{~cm})$ and BF-lh $(7.02 \pm 1.28 \mathrm{~cm})$ than in the ST $(14.87 \pm 3.52 \mathrm{~cm})$ [18]. Because short muscle fiber length generates passive tension early [22,36], SM and BF-lh with shorter muscle fiber length than ST are considered to have been elongated due to earlier generation of passive tension.

The BF-lh is injured during high-speed running, and the SM is injured during dance and kicking [2,3]. The stretching protocol in this study has the effect of decreasing the shear elastic modulus of BF-lh and SM. Thus, reducing the incidence of hamstring strain may be useful. AKE stretching is considered to be useful in clinical situations because it can contribute to a decrease in muscle elasticity without the help of other people when performing stretching for the purpose of reducing muscle elasticity. However, whether reduction of elasticity of muscle after stretching prevents muscle strain is unclear. Investigating whether the protocols of these stretching methods are effective to prevent muscle strain is necessary.

\section{Limitations of the study}

This study has some limitations. First, muscle activity was not measured pre- and post-stretching. However, based on the results of 
previous studies [22,30], the influence of muscle activity is small in the position measured in this study. Secondly, although knee joint extension might not be achieved by contracting the quadriceps muscle, the activity of the quadriceps muscle during stretching was not measured. Furthermore, it is not clear whether the knee joint was maximally extended during AKE stretching. Investigating muscle activity in detail using electromyography is necessary in the future. Thirdly, this study examined the immediate effect of AKE and PKE stretching, but the prolonged effect of stretching was not measured. A previous study showed that muscle stiffness decreased significantly immediately after stretching, but stiffness was reported to have returned to before stretching after 10 to $20 \mathrm{~min}$ [37]. Therefore, further research on the prolonged effects of AKE and PKE stretching is necessary.

\section{Conclusions}

We investigated the acute effect of the difference between AKE and PKE stretching on the shear elastic modulus of the hamstrings. An increase in maximum ROM and a decrease in shear elastic modulus of the hamstrings were noted, but no difference was observed between the procedures. Furthermore, both stretching protocols used in this study are effective for reducing the muscle stiffness of the BF-lh and SM muscles.

\section{Conflict of Interest}

The authors declare that they have no conflict of interest.

\section{References}

[1] Ahmad CS, Redler LH, Ciccotti MG, Maffulli N, Longo UG, Bradley J. Evaluation and management of hamstring injuries. Am J Sports Med 2013; 41: 2933-2947

[2] Askling CM, Tengvar M, Saartok T, Thorstensson A. Acute first-time hamstring strains during high-speed running: a longitudinal study including clinical and magnetic resonance imaging findings. Am J Sports Med 2007; 35: 197-206

[3] Askling CM, Tengvar M, Saartok T, Thorstensson A. Acute first-time hamstring strains during slow-speed stretching: clinical, magnetic resonance imaging, and recovery characteristics. Am J Sports Med 2007; 35: 1716-1724

[4] Crema MD, Guermazi A, Tol JL, Niu J, Hamilton B, Roemer FW. Acute hamstring injury in football players: association between anatomical location and extent of injury-a large single-center MRI report. J Sci Med Sport 2016; 19: 317-322

[5] De Smet AA, Best TM. MR imaging of the distribution and location of acute hamstring injuries in athletes. AJR Am J Roentgenol 2000; 174: 393-399

[6] de Weijer VC, Gorniak GC, Shamus E. The effect of static stretch and warm-up exercise on hamstring length over the course of 24 hours. J Orthop Sports Phys Ther 2003; 33: 727-733

[7] Ekstrand J, Hagglund M, Walden M. Epidemiology of muscle injuries in professional football (soccer). Am J Sports Med 2011; 39: 1226-1232

[8] Ferreira GN, Teixeira-Salmela LF, Guimaraes CQ. Gains in flexibility related to measures of muscular performance: impact of flexibility on muscular performance. Clin J Sport Med 2007; 17: 276-281
[9] Harriss D], Macsween A, Atkinson G. Standards for ethics in sport and exercise science research: 2018 update. Int J Sports Med 2017; 38: $1124-1131$

[10] Hazaki K, Ichihashi N, Morinaga T. Effects of stretching on antagonist muscle following maximal contraction of agonist muscle. Annual Report of the College of Medical. Technology, Kyoto University 1996; 16: $29-35$

[11] Herda T], Costa PB, Walter AA, Ryan ED, Hoge KM, Kerksick CM, Stout JR, Cramer JT. Effects of two modes of static stretching on muscle strength and stiffness. Med Sci Sports Exerc 2011; 43: 1777-1784

[12] Herda T], Herda ND, Costa PB, Walter-Herda AA, Valdez AM, Cramer JT. The effects of dynamic stretching on the passive properties of the muscle-tendon unit. J Sports Sci 2013; 31: 479-487

[13] Hirata K, Miyamoto-Mikami E, Kimura N, Miyamoto N. No association between passive material property and cross-sectional area in human hamstring. J Phys Fitness. Sports Med 2018; 7: 35-40

[14] Hoffren M, Ishikawa M, Komi PV. Age-related neuromuscular function during drop jumps. J Appl Physiol 1985; 103: 1276-1283

[15] Hug F, Tucker K, Gennisson JL, Tanter M, Nordez A. Elastography for muscle biomechanics: toward the estimation of individual muscle force. Exerc Sport Sci Rev 2015; 43: 125-133

[16] Ichihashi N, Umegaki H, Ikezoe T, Nakamura M, Nishishita S, Fujita K, Umehara J, Nakao S, Ibuki S. The effects of a 4-week static stretching programme on the individual muscles comprising the hamstrings. J Sports Sci 2016; 34: 2155-2159

[17] Jackson T], Starkey C, McElhiney D, Domb BG. Epidemiology of hip injuries in the national basketball association: a 24-year overview. Orthop J Sports Med 2013; 1: 2325967113499130

[18] Kellis E, Galanis N, Kapetanos G, Natsis K. Architectural differences between the hamstring muscles. J Electromyogr Kinesiol 2012; 22: 520-526

[19] Komiyama T, Kasai T. Changes in the H-reflexes of ankle extensor and flexor muscles at the initiation of a stepping movement in humans. Brain Res 1997; 766: 227-235

[20] Koo TK, Guo JY, Cohen JH, Parker KJ. Relationship between shear elastic modulus and passive muscle force: an ex-vivo study. J Biomech 2013; 46: 2053-2059

[21] Kot BC, Zhang Z], Lee AW, Leung VY, Fu SN. Elastic modulus of muscle and tendon with shear wave ultrasound elastography: variations with different technical settings. PLoS One 2012; 7: e44348

[22] Le Sant G, Ates F, Brasseur JL, Nordez A. Elastography study of hamstring behaviors during passive stretching. PLoS One 2015; 10: e0139272

[23] Leong HT, Ng GY, Leung VY, Fu SN. Quantitative estimation of muscle shear elastic modulus of the upper trapezius with supersonic shear imaging during arm positioning. PLoS One 2013; 8: e67199

[24] Magnusson SP, Aagaard P, Simonsen EB, Bojsen-Moller F. Passive tensile stress and energy of the human hamstring muscles in vivo. Scand J Med Sci Sports 2000; 10: 351-359

[25] Maisetti O, Hug F, Bouillard K, Nordez A. Characterization of passive elastic properties of the human medial gastrocnemius muscle belly using supersonic shear imaging. J Biomech 2012; 45: 978-984

[26] Marshall PW, Cashman A, Cheema BS. A randomized controlled trial for the effect of passive stretching on measures of hamstring extensibility, passive stiffness, strength, and stretch tolerance. J Sci Med Sport 2011; 14: 535-540

[27] Medeiros DM, Cini A, Sbruzzi G, Lima CS. Influence of static stretching on hamstring flexibility in healthy young adults: systematic review and meta-analysis. Physiother Theory Pract 2016; 32: 438-445

[28] Mendiguchia J, Alentorn-Geli E, Brughelli M. Hamstring strain injuries: are we heading in the right direction? Br J Sports Med 2012; 46: $81-85$ 
[29] Meroni R, Cerri CG, Lanzarini C, Barindelli G, Morte GD, Gessaga V, Cesana GC, De Vito G. Comparison of active stretching technique and static stretching technique on hamstring flexibility. Clin J Sport Med 2010; $20: 8-14$

[30] Miyamoto N, Hirata K, Kanehisa H. Effects of hamstring stretching on passive muscle stiffness vary between hip flexion and knee extension maneuvers. Scand J Med Sci Sports 2017; 27: 99-106

[31] Nakamura M, Ikezoe T, Takeno Y, Ichihashi N. Acute and prolonged effect of static stretching on the passive stiffness of the human gastrocnemius muscle tendon unit in vivo. J Orthop Res 2011; 29: 1759-1763

[32] Nakamura M, Ikezoe T, Takeno Y, Ichihashi N. Time course of changes in passive properties of the gastrocnemius muscle-tendon unit during 5 min of static stretching. Man Ther 2013; 18: 211-215

[33] Nishikawa Y, Aizawa J, Kanemura N, Takahashi T, Hosomi N, Maruyama $\mathrm{H}$ et al. Immediate effect of passive and active stretching on hamstrings flexibility: a single-blinded randomized control trial. J Phys Ther Sci 2015; 27: 3167-3170

[34] O'Sullivan K, Murray E, Sainsbury D. The effect of warm-up, static stretching and dynamic stretching on hamstring flexibility in previously injured subjects. BMC Musculoskelet Disord 2009; 10: 37

[35] Power K, Behm D, Cahill F, Carroll M, Young W. An acute bout static stretching: effects on force and jumping performance. Med Sci Sports Exerc 2004; 36: 1389-1396

[36] Prado LG, Makarenko I, Andresen C, Kruger M, Opitz CA, Linke WA. Isoform diversity of giant proteins in relation to passive and active contractile properties of rabbit skeletal muscles. J Gen Physiol 2005; 126: $461-480$
[37] Ryan ED, Beck TW, Herda T], Hull HR, Hartman M], Costa PB, Defreitas JM, Stout JR, Cramer JT. The time course of musculotendinous stiffness responses following different durations of passive stretching. J Orthop Sports Phys Ther 2008; 38: 632-639

[38] Sekir U, Arabact R, Akova B, Kadagan SM. Acute effects of static and dynamic stretching on leg flexor and extensor isokinetic strength in elite women athletes. Scand J Med Sci Sports 2010; 20: 268-281

[39] Taniguchi K, Shinohara M, Nozaki S, Katayose M. Acute decrease in the stiffness of resting muscle belly due to static stretching. Scand J Med Sci Sports 2015; 25: 32-40

[40] Taylor JL, McCloskey DI. Detection of slow movements imposed at the elbow during active flexion in man. J Physiol 1992; 457: 503-513

[41] Umegaki H, Ikezoe T, Nakamura M, Nishishita S, Kobayashi T, Fujita K et al. Acute effects of static stretching on the hamstrings using shear elastic modulus determined by ultrasound shear wave elastography: Differences in flexibility between hamstring muscle components. Man Ther 2015; 20: 610-613

[42] Winters MV, Blake CG, Trost JS, Marcello-Brinker TB, Lowe LM, Garber MB, Wainner RS. Passive versus active stretching of hip flexor muscles in subjects with limited hip extension: A randomized clinical trial. Phys Ther 2004; 84: 800-807

[43] Woodley S], Mercer SR. Hamstring muscles: Architecture and innervation. Cells Tissues Organs 2005; 179: 125-141

[44] Woods C, Hawkins RD, Maltby S, Hulse M, Thomas A, Hodson A. The Football Association Medical Research Programme: An audit of injuries in professional football-analysis of hamstring injuries. Br J Sports Med 2004; 38: 36-41

\section{Notice}

This article was changed according to the erratum on December 19, 2018.

\section{Erratum}

In the above-mentioned article, the affiliations were corrected as follows:

\section{Gakuto Nakao}

1 Graduate School of Health Sciences, Sapporo Medical University, Sapporo, Japan

2 Department of Rehabilitation, Ebetsu City Hospital, Ebetsu, Japan

\section{Keigo Taniguchi}

3 Department of Physical Therapy, Sapporo Medical

University, Sapporo, Japan

\section{Masaki Katayose}

3 Department of Physical Therapy, Sapporo Medical University, Sapporo, Japan. 